Isabelle KRIER, «"D’une vue oblique”. Écriture et scepticisme dans les Essais », @nalyses, automne 2007

\title{
Isabelle KRIER
}

\section{“D’une vue oblique”. Écriture et scepticisme dans les Essais}

Quelles significations attribuer, dans les Essais de Montaigne, aux inflexions d'allure fortuite vers des propos que rien ne laissait prévoir, aux titres sans rapport explicite avec le contenu des chapitres, à la discontinuité, au désordre? Devons-nous considérer ces « déviances» comme une innovation syntaxique liée à une critique d'un usage dogmatique de la raison et de la parole? Dans Route par ailleurs, André Tournon fait le pari de la littéralité et de la rationalité des Essais.

Optant pour une herméneutique de confiance lucide, il tente de déceler une consistance singulière du texte de Montaigne et les modalités d'expression d'une pensée pyrrhonienne moderne. S'adonnant à une étude "au plus près de la lettre» des Essais, il montre que ce n'est fréquemment que par la voie des anomalies apparentes de composition que peut être sauvegardée la cohérence des chapitres et, par conséquent, leur efficacité. Le scepticisme de Montaigne apparait à ce titre comme un scepticisme affirmatif qui n'exclut ni la conviction ni l'engagement. Le nouveau langage des Essais ne perturbe pas seulement les normes du discours accrédité, il fait subir aussi une mutation radicale à plusieurs notions de base de la philosophie traditionnelle comme celles de différence et d'identité, de condition ou de vie.

En déviant vers des «routes par ailleurs » les itinéraires trop bien reconnus, Montaigne tente de déjouer le piège de l'asservissement de la philosophie aux instances de pouvoir. Son expérience de la parole détournée, dans les pratiques juridiques en vigueur au $\mathrm{XVI}^{\mathrm{e}}$ siècle, lui a montré que même la zététique est susceptible d'instrumentalisation, si elle ne diversifie pas ses opérations. Récusables, gratuits et occasionnels, les propos de Montaigne sont placés sous le signe de l'aléatoire. Mais cette contingence est l'indice d'une authenticité. À une époque où un bouleversement axiologique frappe le droit civil et 
Isabelle KRIER, «"D’une vue oblique”. Écriture et scepticisme dans les Essais », @nalyses, automne 2007

criminel et où un discrédit affecte les témoignages, Montaigne se présente comme un homme d'honneur soucieux de faire entendre sa bonne foi. Dans les Essais, il n'y a plus de menteur parce que la pensée ne se distingue pas de la lettre, elle se fait avec elle.

En proposant un nouveau langage composé de configurations énigmatiques, de perspectives multiples, de discordances, Montaigne devait être conscient du risque d'incompréhension auquel il s'exposait. Qui sont les lecteurs capables de suivre sa parole errante? Charron, Marie de Gournay? André Tournon note que, dans l'édition posthume de 1595, les retouches autographes, l'usage propre à Montaigne des majuscules et des signes de ponctuation ont été souvent négligés. Or, il s'agit là de formes d'expression des « routes par ailleurs ». Après plus de quatre siècles de commentaire des Essais, sommes-nous maintenant susceptibles d'appréhender leur logique particulière et d'entendre leur appel à l'émancipation? La question mérite encore d'être soulevée.

Référence : André Tournon, «Route par ailleurs». Le «nouveau langage» des Essais, Paris, Honoré Champion, coll. «Études montaignistes », 2006, 448 p. 